\title{
System of Potential Barriers in Nanostructures
}

\author{
A. G. Gulyamov ${ }^{1,2}$ \\ ${ }^{1}$ Physic-Technical Institute, Academy of Sciences of Uzbekistan, Tashkent, Uzbekistan \\ ${ }^{2}$ Namangan Engineering Pedagogical Institute, Namangan, Uzbekistan \\ Email: abdurasul.gulyamov@mail.ru
}

Received 23 January 2015; accepted 18 April 2015; published 27 April 2015

Copyright (C 2015 by author and Scientific Research Publishing Inc.

This work is licensed under the Creative Commons Attribution International License (CC BY).

http://creativecommons.org/licenses/by/4.0/

(c) (i) Open Access

\begin{abstract}
Nanoscale superlattice has been investigated theoretically. It has been shown that the deformation effects on the energy spectrum of nanoscale superlattice by changing the interatomic distances as well as varying the width and height of the potential barrier. The potential deformation has been estimated. It has been shown that for different edges of forbidden bands the deformation potential has different values. It has been also analyzed the dependence of the effective mass on energy. It has been determined that the effective mass crosses periodically the zero mark. It has been concluded that this phenomena contributes to the periodic change of the oscillation frequency de Haas-van Alphen effect.
\end{abstract}

\section{Keywords}

Nanoparticle, Potential Barrier, The Potential Well, Kronig-Penney Model, The Deformation Potential

\section{Introduction}

Development of nanotechnology caused increasing requirements of atomic size films. For example, a new lowdimensional nanostructure graphene promises to be one of the key elements of the future nanoelectronics [1]. At present a number of technological methods for obtaining graphene films on flexible solid-state substrates are already developed [2] [3]. Actuality of researches strain-sensing properties of semiconductor films caused by the creation of strain-resistive sensors with semiconductor sensing elements had high sensitivity, reliability, small dimensions and high-technology manufacturing etc.

Under influence of applied voltage film and the substrate should experience different degrees of deformation; however because of they being firm connected each to other the film (due to its small thickness in comparison 
with that substrate) is compressed (or stretched) to conform to the size of the substrate [4]. As a result the system "film-substrate" bends for reaching equilibrium of forces and moments. Thus even a small deformation of the substrate deforms significantly the film grown on it. Under these conditions a potential barriers can appear between the film's nanoparticles. Tunnel and thermionic currents penetrate through such barriers penetrate. Changing distance between the particles of the semiconductor films results in changing of potential barriers and changing band gap of semiconductor. Decision of this problem in the general case is rather difficult and sometimes it is impossible because of absence of sufficient information about parameters of the barriers. Therefore it would be expidient to use the most simplified models for the decision of such a problem. One such of the model is the model of the Kronig-Penney [5]. In this work we tried to apply the model of Kronig-Penney to analyze the effect of deformation on the energy spectrum of semiconductor film.

\section{The Effect of Deformation on the Energy Spectrum of the Superlattice}

The effect of deformation on the tensosensitivity films $\mathrm{Bi}_{2} \mathrm{Te}_{3}$ and $\mathrm{Sb}_{2} \mathrm{Te}_{3}$ obtained by vacuum deposition [6] has been studied in [7]. It has been shown that at small thicknesses of the barriers tensosensitivity these films can take the anomalously large values [7]. However, the effect of deformation on the energy spectrum of the semiconductor film has not been considered.

In the model of Kronig-Penney simple rectangular potential barriers are used [5]. It is known that in this onedimensional model width of wells are equal and they are separated by $a$ potential barriers with thickness $b$ and height $U ; a+b$ is to the period of the superlattice. Solving the Schrödinger's equation we can derive an equation for determination the law of the electrons dispersion in the superlattice:

$$
\frac{\beta^{2}-\alpha^{2}}{2 \alpha \beta} \operatorname{sh}(\beta b) \sin (\alpha a)+\operatorname{ch}(\beta b) \cos (\alpha a)=\cos k(a+b)
$$

Here $\beta=\sqrt{\frac{2 m_{0} E}{\hbar^{2}}}, \alpha=\sqrt{\frac{2 m_{0}(U-E)}{\hbar^{2}}}, \hbar$-Planck's constant, $m_{0}$-the free electron mass, $k$-wave vector, $E$ electron energy, $U$-potential barrier's height. Using Equation (1) can be obtained dependence of the energy $E$ on the wave vector $k$. The influence of the periodic potential depends on the magnitude of this potential as well as the distance between the potential barriers [5].

Thus deforming semiconductor film we act upon its energy spectrum by changing distance between the potential barriers and potential barrier's width $b$.

Knowing how change the forbidden band $\left(\delta E=E_{2}-E_{1}\right)$ and that of the barrier $\left(\delta b=b_{2}-b_{1}\right)$ it will be possible to make an estimation of the deformation potential $\Delta[8]$ :

$$
\Delta=\frac{\delta E}{\varepsilon} .
$$

Here $\varepsilon=\frac{\delta b}{(a+b)}$-the elongation of the superlattice.

\section{Theoretical Calculations}

It should be noted that the calculations allow us to determine only the change of the band gap [9]. Energy shifts of the bands extremums depend on linear expansion $\varepsilon$ linearly and the coefficient of proportionality is called the constant of deformation potential $\delta E_{c}=\Delta_{c} \varepsilon, \delta E_{v}=\varepsilon \Delta_{v}$.

With the help of programme Maple 9.5 the calculated was made and graphs of dependence of wave vector $k$ of the electron's energy $E$ were drawn. At the calculation the following expression was used:

$$
k(E, b)=\frac{\arccos \left(\frac{\beta^{2}-\alpha^{2}}{2 \alpha \beta} \operatorname{sh}(\beta b) \sin (\alpha a)+\operatorname{ch}(\beta b) \cos (\alpha a)\right)}{a+b} .
$$

Let's consider the energy band in which the valence band and conduction bands are shown in Figure 1. One 


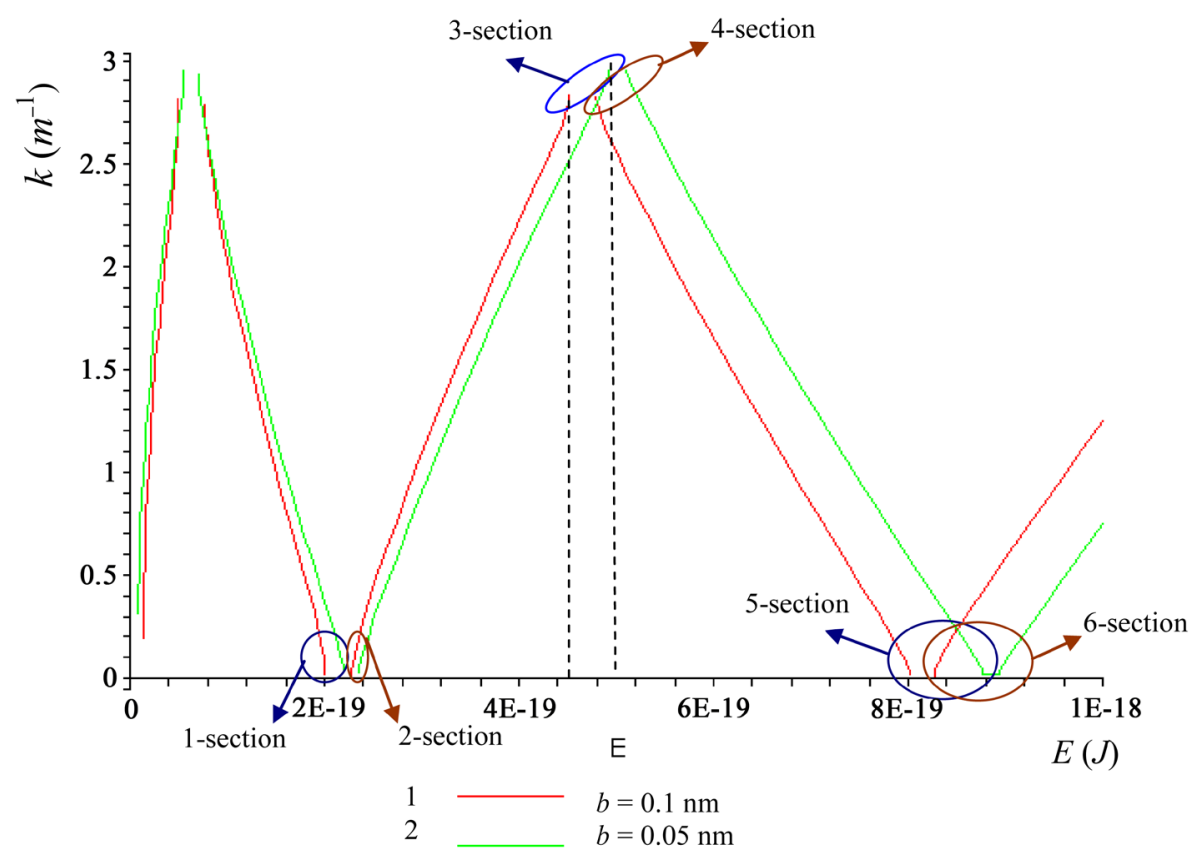

Figure 1. The dependence of the energy of the wave vector for the electronic states for the cases where the thickness of barriers constitute $b=0.1 \mathrm{~nm}$ and $b=0.05 \mathrm{~nm}$.

of the graphs of the dependence of energy on the wave vector for the electronic states at changing of the thickness of the potential barriers is shown there. If the sample is stretched so the relative change of the length defines $\varepsilon$, then the bands shifted slightly as it is indicated on the right of Figure 1.

Basing on the data of Figure 1 and using the expression (2) we can estimate the deformation potential for the third section. At the thickness of the barrier $b_{1}=0.1 \mathrm{~nm}$, the forbidden band's edge $E_{1} \approx 4.42 \times 10^{-19} \mathrm{~J} \approx 2.76 \mathrm{eV}$. If the barrier's thickness $b=0.05 \mathrm{~nm}$, the forbidden band's edge $E_{2} \approx 4.84 \times 10^{-19} \mathrm{~J} \approx 3.03 \mathrm{eV}$. The widths for both cases are the same $a=1 \mathrm{~nm}$. Substituting these values in (2), we get: $\Delta \approx \frac{0.42 \times 10^{-19}}{0.045} \approx 9.3 \times 10^{-19} \mathrm{~J} \approx 5.8 \mathrm{eV}$.

Table 1 shows the values of the deformation potential for each section of Figure 1. Analysis of the data of Table 1 shows that the deformation potential has different values for different band gaps edges. Moreover, as we can see in Figure 1 the left edge of the bands have higher values than the right one.

In addition to the thickness and width of the potential barrier of the potential well another important factor have an influence on the energy spectrum of the sample, that is the potential barrier's height $U$. Analysis of Figure 2 shows that at decreasing the barrier's height gap of the forbidden bands narrow and when the barrier's height is $U=0$, the gaps disappear completely.

The dependence of the effective mass on energy has been also analyzed. The expression has been used for that:

$$
m^{*-1}=\frac{\partial^{2} E}{\hbar^{2} \partial k^{2}}=\frac{\hbar^{2}\left(\frac{\partial f(\beta, a)}{\partial E}\right)}{\left(a^{2} f(\beta, a)+\frac{\left(\frac{\partial^{2} f(\beta, a)}{\partial E^{2}}\right) a^{2}\left(1-f(\beta, a)^{2}\right)}{\left(\frac{\partial f(\beta, a)}{\partial E}\right)^{2}}\right) m_{0}} .
$$

Here $f(\beta, a)=\cos (\beta a) \operatorname{ch}(\gamma b)+\frac{(U-E) \sin (\beta a) \operatorname{sh}(\gamma b)}{2 \sqrt{(U-E) E}}$.

Obtained results are shown in Figure 3. 


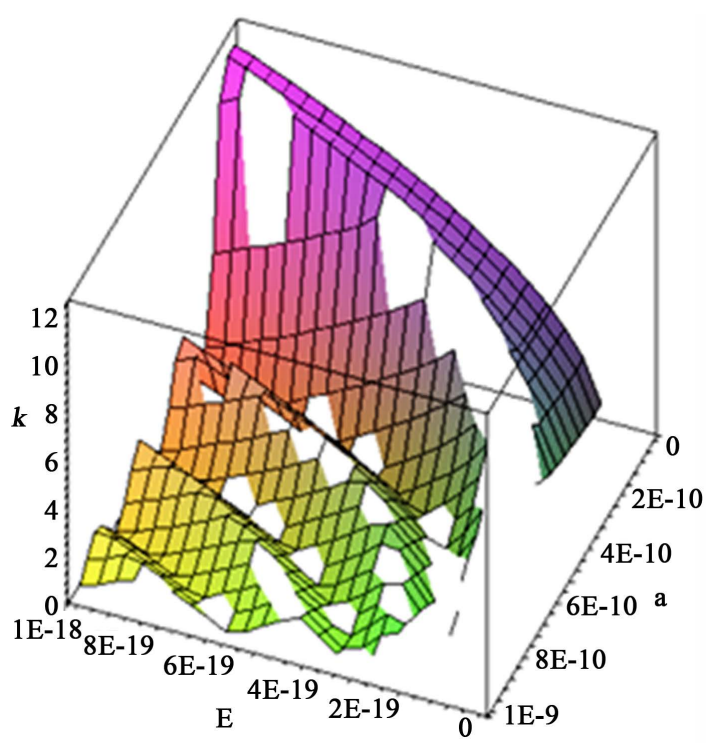

(a)

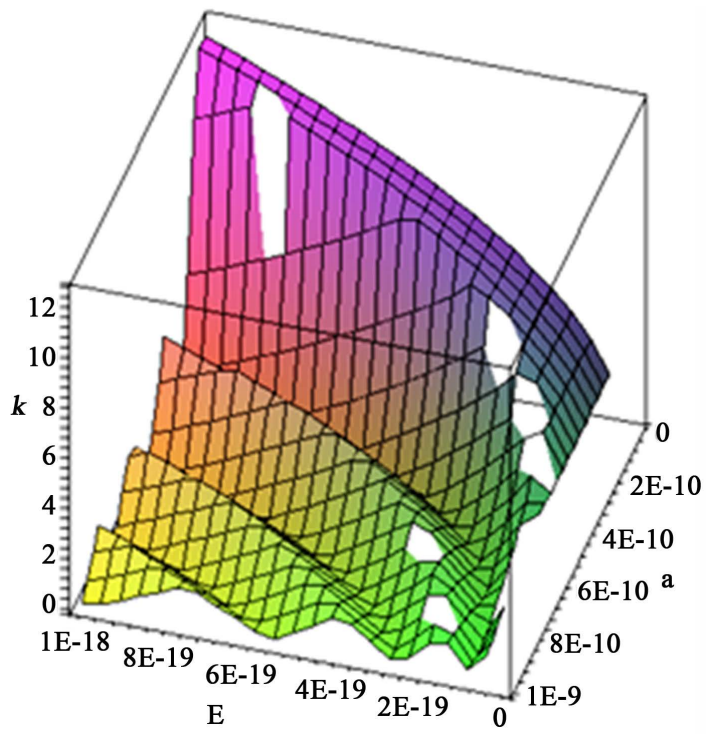

(c)

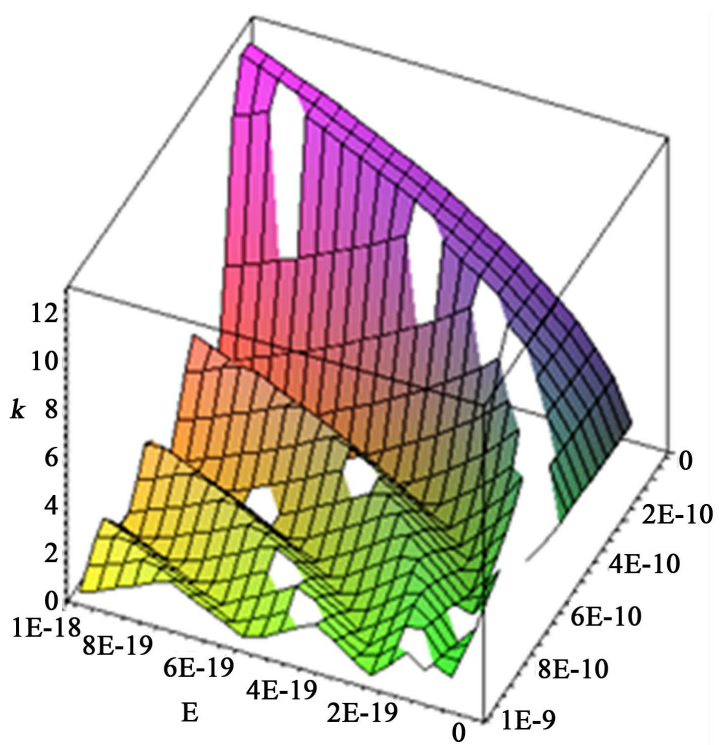

(b)

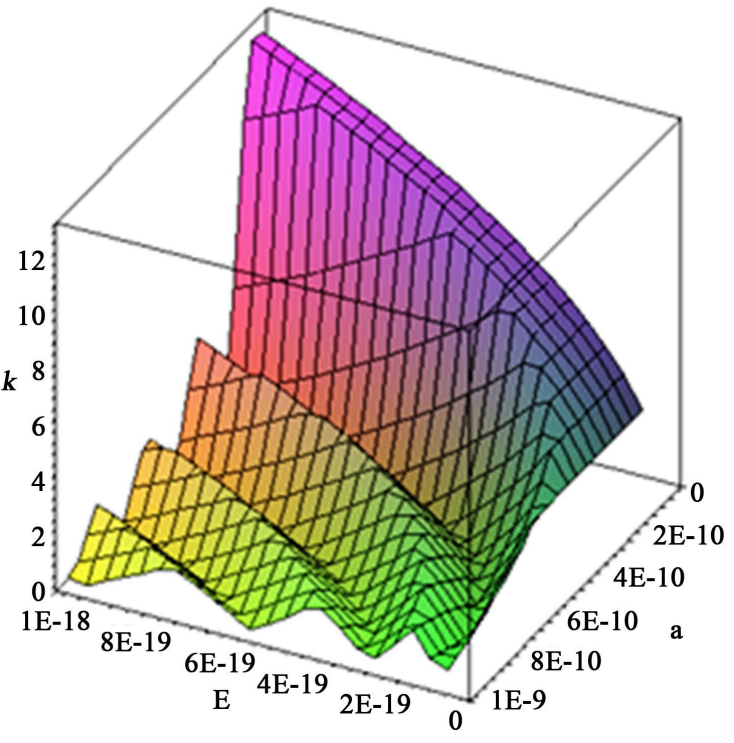

(d)

Figure 2. Three-dimensional plot of the wave vector $k$, the energy $E$ and the width of the potential well of a (for cases (a) $U=1.6 \times 10^{-19}$; (b) $U=0.7 \times 10^{-19}$; (c) $U=0.3 \times 10^{-19}$; (d) $U=0$ ).

Table 1. Analysis of the deformation potential for each section in Figure 1.

\begin{tabular}{|c|c|c|c|c|c|c|}
\hline Number Section & $\mathrm{b}_{1}, \mathrm{~nm}$ & $\mathrm{~b}_{2}, \mathrm{~nm}$ & $\mathrm{a}, \mathrm{nm}$ & $E_{1}, \mathrm{eV}$ & $E_{2}, \mathrm{eV}$ & $\Delta, \mathrm{eV}$ \\
\hline 1-Section & & & & 1.25 & 1.41 & 3.4375 \\
\hline 2-Section & & & & 1.47 & 1.50 & 0.6875 \\
\hline 3-Section & & & & 2.76 & 3.03 & 5.7750 \\
\hline 4-Section & 0.1 & 0.05 & 1 & 3.00 & 3.22 & 4.8125 \\
\hline 5-Section & & & & 5.00 & 5.50 & 11.0000 \\
\hline 6-Section & & & & 5.22 & 5.59 & 8.2500 \\
\hline
\end{tabular}




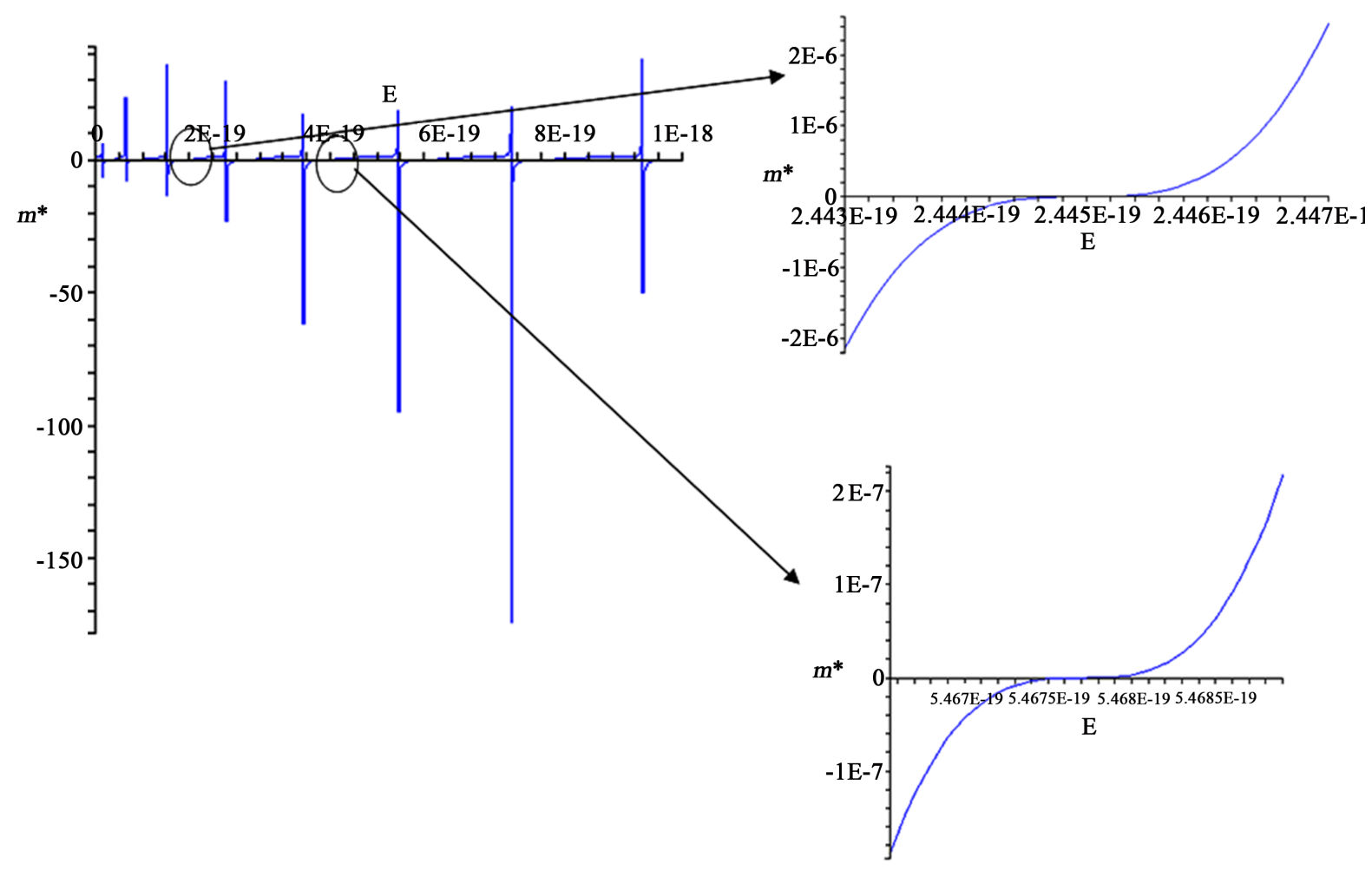

Figure 3. Plot of the effective mass of the energy.

The effect of de Haas-van Alphen (dHvA) in nanostructures of cadmium fluoride was researched in [10]. The authors of [10] found out experimentally periodic change of the oscillation frequency dHvA $\omega_{c}=\frac{e B}{m^{*}}\left(m^{*}-\right.$ effective mass). Figure 3 shows that the effective mass crosses periodically the zero mark. Apparently, it contributes to the periodic change of the oscillation frequency dHvA.

\section{Conclusions}

On the basis of the study it can be concluded that the electron energy spectrum of the potential barriers depends on the barrier's size. Dimensions of the barriers can be changed by the deformation of thin semiconductor films. High tensosensitivity of thin semiconductor films [7] can be explained by the influence of deformation on the size of the potential barriers between the semiconductor's nanoparticles. Anomalously low values of the cyclotron effective mass found in [10] can be caused by the motion of electrons in the potential barriers and wells.

Thus, we can make the following deductions:

-Using dependence of the energy on the wave vector (see Figure 1) obtained on the base of the model Kronig-Penney we can be estimate the deformation potential.

-The deformation potential has different values for different edges of forbidden bands: the left edges of the bands have higher values than the right one (see Figure 1).

-With different widths of the potential barriers $b$ wave vector $k$ has different values, and the value of the wave vector decreases with increasing width of the potential barriers.

-Dependence of the effective mass on energy (shown in Figure 3) explains periodic variation of the oscillation frequency dHvA obtained by authors [10] experimentally.

\section{References}

[1] Novoselov, K.S., et al. (2005) Two-Dimensional Atomic Crystals. Proceedings of the National Academy of Sciences of the United States of America, 102, 10451-10453.

[2] Bae, S., et al. (2010) Roll to Roll Production of 30-Inch Grapheme Inch for Transparent Electrodes. Nature Nanotech- 
nology, 5.

[3] Kim, K.S., Zhao, Y. and Jang, H. (2009) Large-Scale Pattern Growth of Graphene Films for Stretchable Transparent Electrodes. Nature, 457, 706-710. http://dx.doi.org/10.1038/nature07719

[4] Shugurov, A.R. and Panin, A.V. (2009) Mechanisms for Periodic Deformation of the "Film-Substrate" under the Influence of Compressive Stresses. Physical Mesomechanics, 12, 23-32.

[5] Blakemore, J.S. (1988) Solid State Physics. Moscow, Mir.

[6] Shamirzaev, S.H., Yusupova, D.A., Mukhamediev, E.D. and Onarkulov, K.E. (2006) FIP (Fyzicheckaya Injeneriya Poverkhnosti), 4, 86-90.

[7] Gulyamov, G., Gulyamov, A.G. and Majidova, G.N. (2013) Thin-Film Effect in the Potential Barriers in Semiconductor Films. FIP (Fyzicheckaya Injeneriya Poverkhnosti), 11, 243-245.

[8] Kireev, P.S. (1975) Physics of Semiconductors. Vishaya shkola, Moscow.

[9] Harrison, W. (1988) Solid State Theory. Moscow, Mir.

[10] Bagraev, N.T., Brilinskaya, E.S., Danilovsky, E.Yu., Klyachkin, L.E., Malyarenko, A.M. and Romanov, V.V. (2012) FTP, 46, 90-95. 